\title{
Sector retinitis pigmentosa in juvenile nephronophthisis
}

\author{
V. GODEL, A. IAINA, ${ }^{1}$ P. NEMET, AND M. LAZAR \\ From the Department of Ophthalmology, Ichilov Hospital, and the ${ }^{1}$ Department of Nephrology, \\ Sheba Medical Centre, Tel Aviv University Medical School, Israel
}

SUMMARY In a patient with juvenile nephronophthisis, sector retinitis pigmentosa was found as an extrarenal manifestation, establishing a hitherto undescribed variety of retinal degeneration occurring in this disorder. The retinal function in this case was identical with that in the classic type of sector retinitis pigmentosa, namely, subnormal ERG amplitudes but normal cone and rod implicit times. The range of the retinal findings and their autosomal recessive transmission are discussed. Paucity of information makes it difficult to elucidate the basic genetic defect operating in this condition.

Juvenile nephronophthisis (Fanconi et al., 1951) and medullary cystic disease (Smith and Graham, 1945) are hereditary conditions characterised clinically by failure to thrive, anaemia, hyposthenuria, and salt craving, followed by early uraemia. The consensus of most observers is that these diseases are identical, and owing to their clinical and pathological similarities they have been grouped together (Mongeau and Worthen, 1967).

The presence of extrarenal manifestations in juvenile nephronophthisis is obvious (Mainzer et al., 1970; Boichis et al., 1973), and the most frequent allied condition is tapeto-retinal degeneration (Senior et al., 1961). There is ample evidence of such an association in familial cases, and it appears to be due to a pleiotropic effect of the gene involved and not a coincidence of symptoms. A common genetic background for the renal and retinal disorders has been postulated and autosomal recessive inheritance invoked.

We report here a new case of juvenile nephronophthisis which involved a type of retinal degeneration not yet reported in connection with this complex renal disorder.

\section{Case report}

Our interest in this 18-year-old woman was stimulated during routine investigation for possible ocular abnormalities in patients on chronic haemo-

Correspondence to Victor Godel, MD, Department of Ophthalmology, Ichilov Hospital, Tel Aviv, Israel. dialysis. The onset of her renal disorder began 7 years earlier with a normocytic anaemia. Polydipsia and polyuria were observed clinically, and tests of kidney function showed impaired urinary concentrating abilities. Later a high blood urea and serum creatinine were found. A drip intravenous pyelogram showed poor excretion, though there was no anatomical defect. As kidney function deteriorated, a percutaneous kidney biopsy was performed. Findings included scattered medullary cysts and extensive glomerular destruction with interstitial fibrosis and hyalinisation. These histological findings confirmed the clinical suspicion of juvenile nephronophthisis, and the patient was signed up for a haemodialysis programme and future kidney transplant.

She had no visual complaints and was unaware of any ocular disease. An audiogram indicated normal hearing function. She had normal visual acuity and the anterior segments were within normal limits. Ophthalmoscopy disclosed normal optic discs and free maculae. The central parts of the retinal vessels were of normal calibre. Symmetrical bonecorpuscule-like pigment clumps were found in the lower temporal quadrants of both eyes(Fig. 1). These abnormal areas of pigmentation were situated in the region of the equator and anterior to it. The retinal vessels were narrowed and disappeared towards the periphery. The pigment epithelium was atrophied. Colour vision was normal, but in the visual fields large scotomas were found bilaterally in the upper nasal quadrant, corresponding to the retinal lesions

The retinal function profile, investigated by means of electroretinography, showed subnormal ampli- 


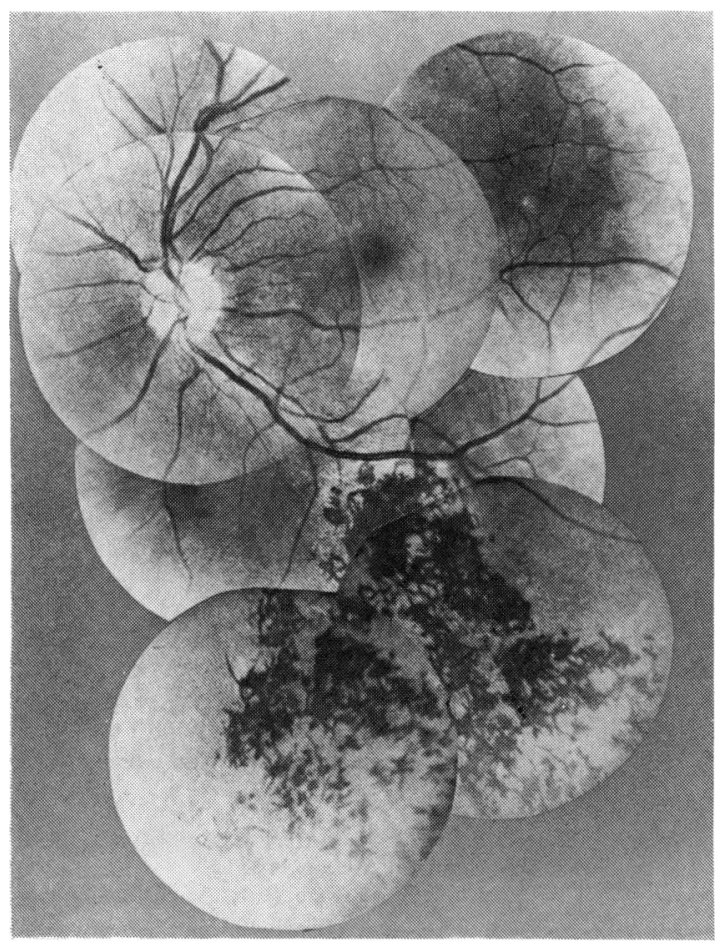

Fig. 1 The fundus of the proband. Note the sectorial distribution of the pigmentary clumps

tudes during the whole process of dark adaptation. There was some increase of the positive ERG amplitudes as a function of stimulus intensity at the steady state of dark adaptation, but they were below the normal values (Fig. 2). Despite the incomplete recovery of the ERG amplitudes, the peak latencies of the positive wave were normal for both photopic and scotopic components. The ERG amplitude response was lower than could be predicted by the ophthalmoscopic retinal appearance.

The family pedigree (Fig. 3) disclosed that the proband had a healthy younger brother and another brother who died at 6 years of age following endstage uraemia. Neither ocular examination nor necropsy was carried out in this case. The parents were first cousins and had not had any significant illnesses. The pregnancies were without problems, and there was no exposure to teratogenic agents. According to the parents the birth weights of the proband and of the deceased child were lower than that of the healthy sib. The parents did not mention any special difficulties in the birth of these children. Inquiry among other family members affirmed that no one showed any symptoms of this syndrome. The parents themselves had normal renal and retinal functions.

\section{Discussion}

The concurrence of the renal and the retinal disorders in our case, known to exist as separate conditions, does recall some observations made in previous cases. Various retinal degenerations have been reported to be associated with juvenile nephronophthisis, and they apparently represent different

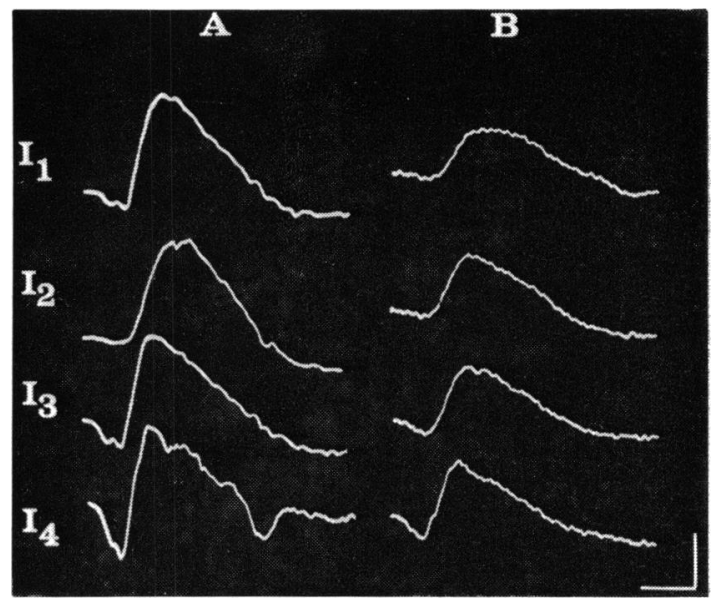

Fig. 2 The electroretinogram of our patient (B) compared with a normal case (A). These electrical responses, after full dark adaptation, were elicited with different intensities (I1-I4). Note the reduced positive amplitude but normal photopic and scotopic implicit times in the index case. Calibration $200 \mu \mathrm{V}, 40 \mathrm{~ms}$

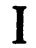

口 O NORMAL MALE AND FAMALE

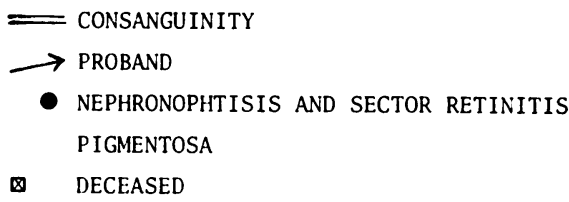

Fig. 3 Family pedigree 
forms of the same disease rather than distinct entities. Among the retinal manifestations of this renal disorder congenital Leber amaurosis (Hussels, 1971; Godel et al., 1978), retinitis pigmentosa (Senior et al., 1961), and central retinitis pigmentosa (Price and Pratt-Johnson, 1970) were described. Our case illustrated a new variety of retinal disease appearing in association with the renal lesions. While such an association may be considered merely fortuitous, evidence has been reported of such relationships between retinitis pigmentosa or its variants and this renal disorder.

There is no argument over the familial nature of this disorder, and the consistency of reported cases leaves little doubt that it is genetically determined. The fact that different retinal disorders occur in different kindreds with juvenile nephronophthisis suggests that a biological mechanism keeps the genes for the syndrome together.

The pedigree of our family supports the interpretation that this disease is due to a recessive gene. Since both parents show no clinical evidence of the disease and they themselves are first cousins, it would seem logical to invoke a recessive pattern of transmission here. Furthermore, affected members of both sexes (the proband and the deceased child) had a normal sib, and there is no known history of other relatives of the parents being so affected.

While there seems little doubt that this genetic syndrome is an autosomal recessive trait, the wide range of retinal anomalies reported suggests there is some variability in the expression of the genetic defect. One might argue that our proband, who presents the typical picture of juvenile nephronophthisis, is affected by an unusual retinal condition which alters the typical expression of the syndrome. This unusual retinal condition, diagnosed by ophthalmoscopy, appeared in the form of typical sector retinitis pigmentosa. Its retinal functional characterisation by electroretinograms showed that the defective scotopic activity was due to a retinal involvement larger than that which could be seen by simple funduscopy. This finding confirms previous reports (Krill et al., 1970) showing that the morphological retinal defect is more widespread than the ophthalmoscopic appearance would suggest.

As regards the temporal aspects of the electroretinograms, normal cone and rod implicit times were found in our case to be in accordance with previously reported findings in this retinal condition (Berson and Howard, 1971).

Although several pedigrees have been reported, no parents have yet been shown convincingly to have renal or retinal changes. In some cases the discovery of a urinary concentrating defect (Pedreira et al., 1968) or electroretinographic changes (Polak et al., 1977) in one of the parents or siblings of affected $\frac{0}{3}$ offsprings has raised the question of a heterozygous 으 state. No such signs of heterozygosity were found $\vec{F}$ in our family.

Unfortunately our family provided no additional information on the nature of the basic genetic $\frac{\bar{c}}{\bar{s}}$ defect in this disease. There are some suggestions $\frac{\vec{\Phi}}{\nabla}$ that the renal involvement is not only genetically $\propto$ determined but might be due to an inborn error of $\omega$ metabolism (Schimke, 1969). Present laboratory $\vec{\circ}$ methods, however, are not sensitive enough to isolate the enzyme abnormalities.

Since the retinal findings in juvenile nephronoph- $\frac{\partial}{\partial}$ thisis take various forms and occur more frequently than has been assumed, careful ophthalmoscopic $\stackrel{\AA}{\perp}$ examination of every such patient would seem to be ? necessary.

\section{References}

Berson, E. L., and Howard, J. (1971). Temporal aspects of the electroretinogram in sector retinitis pigmentosa. Archives of Ophthalmology, 86, 653-665.

Boichis. H., Passwell, J., David, R., and Miller, H. (1973). Congenital hepatic fibrosis and nephronophthisis. Quarterly Journal of Medicine, 165, 221-233.

Fanconi, G., Hanhart, E., von Albertini, A., Euhlinger, R., Dolivo, G., and Prader, A. (1951). Die familiäre juvenile Nephronophthise (die idiopthische parenchymatose Schrumpfniere). Helvetiae Pediatrica Acta, 6, 1-9.

Godel, V., Blumenthal, M., and Iaina, A. (1978). Congenital $\bar{\partial}$ Leber amaurosis, keratoconus and mental retardation in familial juvenile nephronophthisis. Journal of Pediatric $\frac{\mathrm{Q}}{\mathbb{D}}$ Ophthalmology, 15, 89-91.

Hussels, I. E. (1971). Congenital amaurosis and nephronoph- $\overrightarrow{\overrightarrow{0}}$ thisis. Birth Defects, 7, 199-205.

Krill, A. E., Archer, D., and Martin, D. (1970). Sector retinitis pigmentosa. American Journal Ophthalmology, $\frac{\overrightarrow{0}}{0}$ 69, 977-987.

Mainzer, F., Saldino, R. M., Ozonoff, M. B., and Minagi, 흐. H. (1970). Familial nephropathy associated with retinitis $O$ pigmentosa, cerebellar ataxia and skeletal abnormalities. American Journal of Medicine, 49, 556-562.

Mongeau, J. G., and Worthen, H. G. (1967). Nephronophthisis and medullary cystic disease. American Journal of Medicine, 43, 345-355.

Pedreira, F. A., Marmer, E. L., and Bergstrom, W. H. 을 (1968). Familial juvenile nephronophthisis and medullary $D$

cystic disease. Journal of Pediatrics, 73, 77-83.
Polak, B. C. P., Hogewind, B. L., and Van Lith, F. H. M. 主 (1977). Tapetoretinal degeneration associated with recess- N ively inherited medullary cystic disease. American Journal Ophthalmology, 84, 645-651.

Price, J. D. E., and Pratt-Johnson, J. A. (1970). Medullary N cystic disease with degeneration. Canadian Medical Association Journal, 102, 165-167.

Schimke, R. N. (1969). Hereditary renal-retinal dysplasia. Annals of Internal Medicine, 70, 735-744.

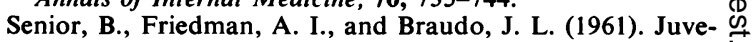
nile familial nephronopathy with tapeto-retinal degeneration: a new oculo-renal dystrophy. American Journal of Ophthalmology, 52, 625-633.

Smith, C. H., and Graham, J. B. (1945). Congenital medullary cysts of the kidney with severe refractory anemia. American Journal of Diseases of Children, 69, 369-377. 